\title{
Solutions of Continuous-Time Preview Two-Player Differential Games
}

\author{
Wen-Hou $\mathrm{Ma}^{1}$ and Huei Peng 2 \\ Department of Mechanical Engineering and Applied Mechanics \\ University of Michigan \\ Ann Arbor, MI 48109-2125 \\ hpeng@umich.edu
}

\begin{abstract}
The continuous-time two-player preview game problems are studied in this paper. A preview game problem is formulated when the input signals generated by one of the two players are delayed (e.g., by actuator dynamics), and are previewed by the other player. In accordance with the control terminology, these two players are referred to as the control player and the disturbance player, respectively. Both control-advantaged and disturbance-advantaged game solutions are presented. When the solution of an infinite horizon problem exists, the response of the closed-loop system can be analyzed in the frequency domain. A vehicle handling problem is used as an example for the analysis. It is shown that the preview action of the advantaged player effectively reduces the authority of the disadvantaged player, and results in significantly improved performance compared with feedback-only game algorithms.
\end{abstract}

\section{INTRODUCTION}

Game theory studies the optimal strategies for players involved in multi-person interactions. The work by von Neumann and Morgenstern (1947) and Kuhn (1953) laid the foundation of static games. Essentially, to solve static games, all possible decisions and consequences are laid out, and the game evolves according to a tree structure. The problem becomes a dynamic game when the controlled process is described by differential or difference equations. Concepts and techniques developed for the optimal control theory (which address one-player "games") were borrowed and incorporated into the multi-player optimal solutions.

Game theories were soon recognized to be useful in the design of robust controllers (e.g., Dorato and Drenick 1964, Witsenhausen 1968, Yaesh and Shaked 1989). The resulting control algorithm is robust in the sense that it achieves "minimax" performance, i.e., the performance is optimized for the worst possible disturbances. When the performance index is quadratic, the non-cooperative dynamic game problem for linear time invariant systems over infinite time horizon was found to be equivalent to an $H_{\infty}$ problem (Basar and Bernhard 1995). In fact, the $H_{\infty}$ control solution was first solved in time domain using the game theory (Mageirou and Ho 1977), before the solution in frequency domain was obtained.

It has been recognized that in formulating a game problem, one of the most crucial steps is to describe the conflicting situations and actions clearly. Information structure (i.e. what one

\footnotetext{
${ }^{1}$ Mechanical Dynamics Inc.

2 Assistant Professor
} 
player knows relative to others) was found to be especially important. Solutions under several information structures (e.g. open-loop, closed-loop with perfect state, 1-step delay, closed-loop with imperfect state measurement) have been obtained (see Basar and Bernhard 1995). The cases when preview information is available to one of the players, however, have not been solved. Preview game problems frequently are the result of actuator delays. When delays are present, a game problem can be solved in the discrete-time domain by augmenting the plant dynamics with the delayed inputs. However, the order of the system may become very high. An alternative approach, which is suggested in this paper, is to solve the preview game problem while maintaining the system order. The main advantage of including preview action in the design process is that the control (or disturbance) algorithm will rely partly on the feedforward information rather than solely on feedback gains. This is important because, in order to render a solution, the "gains" of the disturbance player is usually limited. With a feedforward component, it will be possible to obtain a stronger disturbance player. This is important for applications such as "worst-case disturbance generation" recently proposed (Ma and Peng 1996). Both controladvantaged and disturbance-advantaged preview game problems will be presented. The basic ideas in solving the preview game theory are exactly the same as the preview LQ control theory (Tomizuka and Whitney 1975, Tomizuka 1976), with minor modification to fit into the twoplayer framework.

The remainder of this paper is organized as follows: Section 2 contains the solution of the original non-cooperative 2-player game problems. The preview game problem is officially defined in Section 3, the solution of which is presented in Section 4. Frequency domain analysis results of an example system are shown in section 5. Finally, conclusions are drawn in Section 6.

\section{TWO-PLAYER GAME THEORY SOLUTION (2P)}

The plant studied in this standard non-cooperative two player game problem is assumed to be linear, time invariant and has the following state space representation:

$$
\begin{aligned}
& \underline{\dot{x}}(t)=A \underline{x}(t)+B \underline{u}(t)+G \underline{w}(t) \\
& y(t)=C \underline{x}(t)+D_{u} \underline{u}(t)+D_{w} \underline{w}(t)
\end{aligned}
$$

where $\underline{u}(t)$ is the control vector and $\underline{w}(t)$ is the disturbance vector. These two time functions have contradicting objective, in the sense that $\underline{u}(t)$ (the control) tries to minimize, while $\underline{w}(t)$ (the disturbance) tries to maximize the following cost function:

$$
J(t)=\frac{1}{2} \int_{t}^{t_{f}} \underline{x}^{T}(t) Q \underline{x}(t)+\underline{u}^{T}(t) R \underline{u}(t)-\underline{w}^{T}(t) P \underline{w}(t) d t
$$

where $\mathrm{Q}, \mathrm{R}$, and $\mathrm{P}$ are weighting matrices that are positive semi-definite $(\mathrm{Q})$ and positive definite ( $\mathrm{R}$ and $\mathrm{P}$ ), respectively. The finite-horizon solution of this two-player game is well documented 
in the literature (e.g., Basar and Bernhard 1995). The solutions for the perfect information case are summarized below. The optimal control and disturbance laws are

$$
\begin{aligned}
& \underline{w}^{*}(t)=P^{-1} G^{T} K(t) \underline{x}(t) \\
& \underline{u}^{*}(t)=-R^{-1} B^{T} K(t) \underline{x}(t)
\end{aligned}
$$

respectively, where the gain matrix $\mathrm{K}$ is solved from the game Riccati equation

$$
\begin{aligned}
& \dot{K}(t)=-A^{T} K(t)-K(t) A+K(t)\left(B R^{-1} B^{T}-G P^{-1} G^{T}\right) K(t)-Q \\
& K\left(t_{f}\right)=0
\end{aligned}
$$

The infinite horizon solution of Eq.(4) does not always exist. A sufficient condition for the solution of the Algebraic Riccati Equation to exist is stated in Basar and Bernhard (1995, Theorem 9.7). In Section 5, when we apply Eq.(3) as time-invariant state feedback schemes, we imply that the weighting matrix $\mathrm{P}$ has been selected carefully such that this sufficient condition is satisfied.

\section{PROBLEM STATEMENT}

When significant actuation delays exist for one of the players, the structure of the plant dynamics (Eq.(1)) stays unchanged. However, a new game problem needs to be formulated which involves solving the future actions of the delayed player (see Figure 1). The cost function (Eq.(2)) also need to be modified slightly under the new information structure, but is assumed to maintain the quadratic form shown in Eq.(2). For the control advantaged case, for example, the game problem is to find the optimal strategy of the minimizer $(u(t))$ and the maximizer $\left(w\left(t+t_{l a}\right)\right)$ so that the cost function

$$
J\left(t_{o}\right)=\frac{1}{2} \int_{t_{0}}^{t_{f}}\left[x^{T}(t) Q(t) x(t)+u^{T}(t) R(t) u(t)-w^{T}\left(t+t_{l a}\right) P(t) w\left(t+t_{l a}\right)\right] d t
$$

is optimized. The disturbance advantaged case is defined as an exact dual problem and the details will be presented later in Section 4.2.

\section{PREVIEW TWO-PLAYER GAME SOLUTIONS}

\subsection{Control Advantaged Information Structure (2PPU)}

In this case, significant delays are assumed to exist in the disturbance channel. At time $t$, the control player has access to the disturbance signal within a finite preview window $w(t+\tau)$, $0 \leq \tau \leq t_{l a}$, where $t_{l a}$ is the delay time. The cost function shown in Eq.(5) is to be optimized. From the Principle of optimality, we have

$$
0=\min _{u(t)} \max _{w\left(t+t_{l a}\right)}\left\{\frac{1}{2}\left[x^{T}(t) Q(t) x(t)+u^{T}(t) R(t) u(t)-w^{T}\left(t+t_{l a}\right) P(t) w\left(t+t_{l a}\right)\right]+\frac{d J^{*}(t)}{d t}\right\}
$$

Define the Hamiltonian function 


$$
H \equiv \frac{1}{2}\left[x^{T}(t) Q(t) x(t)+u^{T}(t) R(t) u(t)-w^{T}\left(t+t_{l a}\right) P(t) w\left(t+t_{l a}\right)\right]+\frac{d J^{*}(t)}{d t}
$$

we could obtain

$$
\begin{aligned}
& \left.\frac{\partial H}{\partial u(t)}\right|_{u^{*}(t)}=0 \Rightarrow u^{*}(t)=-R^{-1}(t) \frac{\partial}{\partial u(t)}\left(\frac{d J^{*}}{d t}\right)^{T} \\
& \left.\frac{\partial H}{\partial w\left(t+t_{l a}\right)}\right|_{w^{*}\left(t+t_{l a}\right)}=0 \Rightarrow w^{*}\left(t+t_{l a}\right)=P^{-1}(t) \frac{\partial}{\partial w\left(t+t_{l a}\right)}\left(\frac{d J^{*}}{d t}\right)^{T}
\end{aligned}
$$

The optimal cost is assumed to be approximated by the following generalized quadratic function

$$
\begin{aligned}
J^{*}(t) & =\frac{1}{2} x(t)^{T} K(t) x(t)+\frac{1}{2} \int_{0}^{t_{l a}} \int_{0}^{t_{l a}} w^{T}\left(t+l_{1}\right) K_{w}\left(t, l_{1}, l_{2}\right) w\left(t+l_{2}\right) d l_{1} d l_{2} \\
& +x(t)^{T} \int_{0}^{t_{l a}} F_{1}(t, l) w(t+l) d l
\end{aligned}
$$

Therefore, we have

$$
\frac{\partial J^{*}}{\partial x}=x(t)^{T} K(t)+\int_{0}^{t_{a}} w^{T}(t+l) F_{1}^{T}(t, l) d l
$$

and

$$
\begin{aligned}
\frac{d J^{*}}{d t} & =\frac{1}{2}\left[\dot{x}(t)^{T} K(t) x(t)+x^{T} K^{\prime}(t) x(t)+x^{T} K(t) \dot{x}(t)\right] \\
& +\frac{1}{2} \int_{0}^{t_{l a}} \int_{0}^{t_{l a}}\left[\frac{\partial w^{T}\left(t+l_{1}\right)}{\partial t} K_{w}\left(t, l_{1}, l_{2}\right) w\left(t+l_{2}\right)+w^{T}\left(t+l_{1}\right) \frac{\partial K_{w}\left(t, l_{1}, l_{2}\right)}{\partial t} w\left(t+l_{2}\right)\right. \\
& \left.+w^{T}\left(t+l_{1}\right) K_{w}\left(t, l_{1}, l_{2}\right) \frac{\partial w\left(t+l_{2}\right)}{\partial t}\right] d l_{1} d l_{2} \\
& +x^{T} \int_{0}^{t_{l a}} F_{1}(t, l) w(t+l) d l+x^{T} \int_{0}^{t_{l a}}\left[\frac{\partial F_{1}(t, l)}{\partial t} w(t+l)+F_{1}(t, l) \frac{\partial w(t+l)}{\partial t}\right] d l
\end{aligned}
$$

Substitute Eqs.(10) and (11) into Eqs.(7) and (8), we obtain

$$
\begin{aligned}
& u^{*}(t)=-R^{-1}(t) B^{T}\left[K(t) x(t)+\int_{0}^{t_{l a}} F_{1}(t, l) w(t+l) d l\right] \\
& w^{*}\left(t+t_{l a}\right)=P^{-1}(t) F_{1}^{T}\left(t, t_{l a}\right) x(t)
\end{aligned}
$$

which are the optimal control and disturbance laws for this 2PPU game. From Eqs.(6), (12) and (13), we have

$$
\begin{aligned}
& \frac{1}{2} x^{T} Q x+\frac{1}{2}\left\{R^{-1} B^{T}\left[K x+\int_{0}^{t_{l a}} F_{1}(t, l) w(t+l) d l\right)\right]^{T} R\left\{R^{-1} B^{T}\left[K x+\int_{0}^{t_{l a}} F_{1}(t, l) w(t+l) d l\right)\right] \\
& -\frac{1}{2}\left(P^{-1} F_{1}^{T}\left(t, t_{l a}\right) x\right)^{T} P\left(P^{-1} F_{1}^{T}\left(t, t_{l a}\right) x\right)+\frac{1}{2}(A x+B u+G w)^{T} K x+\frac{1}{2} x^{T} K^{\prime} x+\frac{1}{2} x^{T} K(A x+B u+G w) \\
& +\frac{1}{2} \int_{0}^{t_{a}} \int_{0}^{t_{l a}}\left[\frac{\partial w^{T}\left(t, l_{1}\right)}{\partial t} K_{w}\left(t, l_{1}, l_{2}\right) w\left(t+l_{2}\right)+w^{T}\left(t+l_{1}\right) \frac{\partial K_{w}\left(t, l_{1}, l_{2}\right)}{\partial t} w\left(t+l_{2}\right)\right. \\
& \left.+w^{T}\left(t+l_{1}\right) K_{w}\left(t, l_{1}, l_{2}\right) \frac{\partial w\left(t+l_{2}\right)}{\partial t}\right] d l_{1} d l_{2} \\
& \left.+(A x+B u+G w)^{T} \int_{0}^{t_{l a}} F_{1}(t, l) w(t+l) d l\right)+x^{T} F_{1}\left(t, t_{l a}\right) w\left(t+t_{l a}\right)-x^{T} F_{1}(t, 0) w(t) \\
& -x^{T} \int_{0}^{t_{l a}} \frac{\partial F_{1}(t, l)}{\partial} w(t+l) d l+x^{T} \int_{0}^{t_{l a}} \frac{\partial F_{1}(t, l)}{\partial t} w(t+l) d l=0
\end{aligned}
$$

Note the following two facts: 


$$
\begin{aligned}
& \int_{0}^{t_{l a}} \int_{0}^{t_{l a}} \frac{\partial w^{T}\left(t+l_{1}\right)}{\partial t} K_{w}\left(t, l_{1}, l_{2}\right) w\left(t+l_{2}\right) d l_{1} d l_{2} \\
= & \int_{0}^{t_{l a}}\left[w^{T}\left(t+t_{l a}\right) K_{w}\left(t, t_{l a}, l\right) w(t+l)-w^{T}(t) K_{w}(t, 0, l) w(t+l)\right] d l \\
- & \int_{0}^{t_{l a}} \int_{0}^{t_{l a}} w^{T}\left(t+l_{1}\right) \frac{\partial K_{w}\left(t, l_{1}, l_{2}\right)}{\partial l_{1}} w\left(t+l_{2}\right) d l_{1} d l_{2}
\end{aligned}
$$

and

$$
\begin{aligned}
& \int_{0}^{t_{l a}} \int_{0}^{t_{l a}} w^{T}\left(t+l_{1}\right) K_{w}\left(t, l_{1}, l_{2}\right) \frac{\partial w\left(t+l_{2}\right)}{\partial t} d l_{1} d l_{2} \\
= & \int_{0}^{t_{l a}}\left[w^{T}(t+l) K_{w}\left(t, l, t_{l a}\right) w\left(t+t_{l a}\right)-w^{T}(t+l) K_{w}(t, l, 0) w(t)\right] d l \\
- & \int_{0}^{t_{l a}} \int_{0}^{t_{l a}} w^{T}\left(t+l_{1}\right) \frac{\partial K_{w}\left(t, l_{1}, l_{2}\right)}{\partial l_{2}} w\left(t+l_{2}\right) d l_{1} d l_{2}
\end{aligned}
$$

We obtain, by grouping similar terms in Eq.(14),

$$
\begin{aligned}
& \dot{K}(t)=-A^{T} K(t)-K(t) A+K(t) B R^{-1}(t) B^{T} K(t)-F_{1}\left(t, t_{l a}\right) P^{-1}(t) F_{1}^{T}\left(t, t_{l a}\right)-Q(t) \\
& K\left(t_{f}\right)=0 \\
& \frac{\partial F_{1}(t, l)}{\partial t}=\frac{\partial F_{1}(t, l)}{\partial l}+\left(K(t) B R^{-1}(t) B^{T}-A^{T}\right) F_{1}(t, l)-F_{1}\left(t, t_{l a}\right) P^{-1}(t) K_{w}\left(t, t_{l a}, l\right) \\
& F_{1}(t, 0)=K(t) G \\
& \frac{\partial K_{w}\left(t, l_{1}, l_{2}\right)}{\partial t}=\frac{\partial K_{w}\left(t, l_{1}, l_{2}\right)}{\partial l_{1}}+\frac{\partial K_{w}\left(t, l_{1}, l_{2}\right)}{\partial l_{2}}+F_{1}^{T}\left(t, l_{1}\right) B R^{-1}(t) B^{T} F_{1}\left(t, l_{2}\right) \\
& K_{w}(t, 0, l)=G^{T} F_{1}(t, l)
\end{aligned}
$$

When $(A, B)$ is stabilizable and $\left(A, Q^{1 / 2}\right)$ is detectable, it is well known that a solution of the (generalized) Algebraic Riccati Equation

$$
A^{T} K+K A-K B R^{-1} B^{T} K+F_{1}\left(t_{l a}\right) P^{-1} F_{1}^{T}\left(t_{l a}\right)+Q=0
$$

exists when the P matrix is large enough (Basar and Bernhard 1995). Eq.(18) needs to be solved simultaneously with the time-invariant versions of Eqs.(16) and (17), which are

$$
\begin{aligned}
& 0=\frac{d F_{1}(l)}{d l}+\left(K B R^{-1} B^{T}-A^{T}\right) F_{1}(l)-F_{1}\left(t_{l a}\right) P^{-1} K_{w}\left(t_{l a}, l\right) \\
& F_{1}(0)=K G \\
& 0=\frac{\partial K_{w}\left(l_{1}, l_{2}\right)}{\partial l_{1}}+\frac{\partial K_{w}\left(l_{1}, l_{2}\right)}{\partial l_{2}}+F_{1}^{T}\left(l_{1}\right) B R^{-1} B^{T} F_{1}\left(l_{2}\right) \\
& K_{w}(0, l)=G^{T} F_{1}(l)
\end{aligned}
$$

respectively. It is interesting to note that Eq.(15) reduces to Eq.(4) when the preview time becomes zero.

\subsection{Disturbance Advantaged Information Structure (2PPW)}


Under this information structure, the action of the control player is delayed and the disturbance player can preview the control signal in a finite preview window (see Figure 1(b)). More precisely, at time $t$, the disturbance generator has access to the control signals between time $t$ and $t+t_{l a}$. This problem is an exact dual of the problem presented in section 4.1. Therefore, intermediate steps are omitted and only crucial equations are presented below. The cost function for this problem is

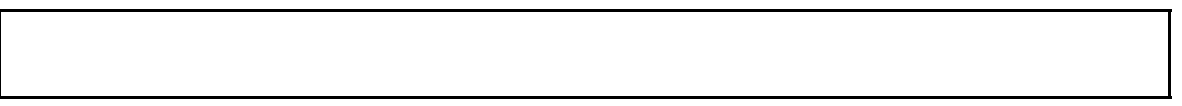

Following the same procedure shown in section 4.1, we obtain

$$
\begin{aligned}
& u^{*}\left(t+t_{d}\right)=-R^{-1}(t) F_{1}^{T}\left(t, t_{l a}\right) x(t) \\
& w^{*}(t)=P^{-1}(t) G^{T}\left[K(t) x(t)+\int_{0}^{t_{l a}} F_{1}(t, l) u(t+l) d l\right]
\end{aligned}
$$

where the feedback and preview gains are solved from the coupled equations

$$
\begin{aligned}
& \dot{K}(t)+A^{T} K(t)+K(t) A+K(t) G P^{-1}(t) G^{T} K(t)-F_{1}\left(t, t_{l a}\right) R^{-1}(t) F_{1}^{T}\left(t, t_{l a}\right)+Q(t)=0 \\
& K\left(t_{f}\right)=0 \\
& \frac{\partial F_{1}(t, l)}{\partial t}=\frac{\partial F_{1}(t, l)}{\partial l}-\left(K(t) G P^{-1}(t) G^{T}+A^{T}\right) F_{1}(t, l)+F_{1}\left(t, t_{l a}\right) R^{-1}(t) K_{u}\left(t, t_{l a}, l\right) \\
& F_{1}(t, 0)=K(t) B \\
& \frac{\partial K_{u}\left(t, l_{1}, l_{2}\right)}{\partial t}=\frac{\partial K_{u}\left(t, l_{1}, l_{2}\right)}{\partial l 1}+\frac{\partial K_{u}\left(t, l_{1}, l_{2}\right)}{\partial l 2}-F_{1}^{T}\left(t, l_{1}\right) G P^{-1}(t) G^{T} F_{1}\left(t, l_{2}\right) \\
& K_{u}(t, 0, l)=B^{T} F_{1}(t, l)
\end{aligned}
$$

For infinite horizon problems, Eqs.(23)-(25) reduce to

$$
\begin{aligned}
& A^{T} K+K A+K G P^{-1} G^{T} K-F_{1}\left(t_{l a}\right) R^{-1} F_{1}^{T}\left(t_{l a}\right)+Q=0 \\
& 0=\frac{d F_{1}(l)}{d l}-\left(K G P^{-1} G^{T}+A^{T}\right) F_{1}(l)+F_{1}\left(t_{l a}\right) R^{-1} K_{u}\left(t_{l a}, l\right) \\
& F_{1}(0)=K B
\end{aligned}
$$

and

$$
\begin{aligned}
& 0=\frac{\partial K_{u}\left(l_{1}, l_{2}\right)}{\partial l_{1}}+\frac{\partial K_{u}\left(l_{1}, l_{2}\right)}{\partial l_{2}}-F_{1}^{T}\left(l_{1}\right) G P^{-1} G^{T} F_{1}\left(l_{2}\right) \\
& K_{u}(0, l)=B^{T} F_{1}(l)
\end{aligned}
$$

Again, Eq.(23) reduces to Eq.(4) when the preview time becomes zero.

\section{Frequency Domain Analysis}

In this section, the transfer functions of all the three two-player game cases presented in this paper are derived. Obviously, solutions of the algebraic equations need to be used to obtain the transfer functions.

\subsection{Non-previewed Case (2P)}


Let $K_{s s}$ to denote the steady-state solution of Eq.(4). From Eqs.(1) and (3), it is straightforward to obtain the transfer function from the control signal $\mathrm{u}(\mathrm{t})$ to the output $\mathrm{y}(\mathrm{t})$ :

$$
G_{u \rightarrow y}(s)=\left(C+D_{w} P^{-1} G^{T} K_{s s}\right)\left(s I-A-G P^{-1} G^{T} K_{s s}\right)^{-1} B+D_{u}
$$

\subsection{Control Advantaged Case (2PPU)}

For infinite-horizon problems, both feedback and preview gains become time-invariant. Therefore, Eq.(13) can be rewritten as $W^{*}(s)=P^{-1} F_{1}^{T}\left(t_{l a}\right) X(s) e^{-t_{l a} s}$, where $F_{1}\left(t_{l a}\right)$ is the terminal solution of Eq.(19). Substitute this optimal disturbance law into Eqs.(1) and (3), we obtain

$$
G_{u \rightarrow y}(s)=\left(C+D_{w} P^{-1} F_{1}^{T}\left(t_{l a}\right) e^{-t_{l a} s}\right)\left(s I-A-G P^{-1} F_{1}^{T}\left(t_{l a}\right) e^{-t_{l a} s}\right)^{-1} B+D_{u}
$$

\subsection{Disturbance Advantaged Case (2PPW)}

From Eq.(22), the optimal disturbance can be written in the frequency domain as

$$
W^{*}(s)=P^{-1} G^{T}\left[K_{s s w} X(s)+\left(\int_{0}^{t_{l a}} F_{1}(l) e^{l s} d l\right) U(s)\right]
$$

where $K_{s s w}$ and $F_{1}(l)$ are the solutions of Eqs.(26) and (27), respectively. The closed-loop dynamics thus are

$$
\begin{aligned}
& s X(s)=A X(s)+B U(s)+G P^{-1} G^{T} K_{s s w} X(s)+\left(G P^{-1} G^{T} \int_{0}^{t_{l a}} F_{1}(l) e^{l s} d l\right) U(s) \\
\Rightarrow & X(s)=\left(s I-A-G P^{-1} G^{T} K_{s s w}\right)^{-1}\left(B+G P^{-1} G^{T} \int_{0}^{t_{l a}} F_{1}(l) e^{l s} d l\right) U(s)
\end{aligned}
$$

Therefore, the transfer function from $\mathrm{u}(\mathrm{t})$ to the output $\mathrm{y}(\mathrm{t})$ is

$$
\begin{aligned}
G_{u \rightarrow y}(s) & =\left(C+D_{w} P^{-1} G^{T} K_{s s w}\right)\left(s I-A-G P^{-1} G^{T} K_{s s w}\right)^{-1}\left(B+G P^{-1} G^{T} \int_{0}^{t_{l a}} F_{1}(l) e^{l s} d l\right) \\
& +D_{u}+D_{w} P^{-1} G^{T} \int_{0}^{t_{l a}} F_{1}(l) e^{l s} d l
\end{aligned}
$$

\subsection{Frequency Response}

The frequency domain analysis of a vehicle steering control system is presented below. The vehicle lateral dynamics is assumed to be described by the following fourth order model (Fenton et al. 1976):

$$
\frac{d}{d t}\left\{\begin{array}{c}
y_{r} \\
\dot{y}_{r} \\
\psi-\psi_{d} \\
\dot{\psi}-\dot{\psi}_{d}
\end{array}\right\}=\left[\begin{array}{cccc}
0 & 1 & 0 & 0 \\
0 & \frac{A_{1}}{u_{0}} & -A_{1} & \frac{A_{2}}{u_{0}} \\
0 & 0 & 0 & 1 \\
0 & \frac{A_{3}}{u_{0}} & -A_{3} & \frac{A_{4}}{u_{0}}
\end{array}\right]\left\{\begin{array}{c}
y_{r} \\
\dot{y}_{r} \\
\psi-\psi_{d} \\
\dot{\psi}-\dot{\psi}_{d}
\end{array}\right\}+\left\{\begin{array}{c}
0 \\
B_{1} \\
0 \\
B_{2}
\end{array}\right\} \delta(t)+\left\{\begin{array}{c}
0 \\
A_{2}-u_{0}{ }^{2} \\
0 \\
A_{4}
\end{array}\right\} w(t)
$$

where $y_{r}$ is the lateral deviation of the mass center of the vehicle from the reference (e.g. lane center), $\psi$ is the yaw angle, $\psi_{d}$ is the desired yaw angle obtained from the road reference, and $u_{o}$ is the vehicle speed, which is assumed to be constant. $\delta(t)$ is the control (front steering 
angle) and $\mathrm{w}(\mathrm{t})$ is the disturbance (curvature of the road). $A_{i}$ and $B_{i}$ depend on vehicle parameters and are known to be (Peng and Tomizuka 1990)

$$
\begin{array}{lll}
A_{1}=\frac{\left(C_{\alpha f}+C_{\alpha r}\right)}{m} \quad A_{2}=\frac{\left(C_{\alpha r} a-C_{\alpha f} b\right)}{m} & A_{3}=\frac{\left(C_{\alpha r} a-C_{\alpha f} b\right)}{I_{z}} \\
A_{4}=\frac{\left(C_{\alpha f} a^{2}+C_{\alpha r} b^{2}\right)}{I_{z}} & B_{1}=\frac{F_{x}-C_{\alpha f}}{m} & B_{2}=\frac{a\left(F_{x}-C_{\alpha f}\right)}{I_{z}}
\end{array}
$$

The vehicle parameters used in the simulations are listed in Table 1. In this preview game problem, the control player (steering) tries to minimize, while the disturbance player (road curvature) tries to maximize the vehicle lateral tracking error and lateral acceleration. The tracking error is assumed to be measured at a distance, $\mathrm{d}_{\mathrm{s}}$, ahead of the vehicle c.g.

Table 1 Nominal values of the vehicle parameters

\begin{tabular}{|l|c|c|c|c|c|c|}
\hline Parameter & $\mathrm{m}(\mathrm{kg})$ & $\mathrm{Iz}(\mathrm{kg}-\mathrm{m} 2)$ & $C_{\alpha}(\mathrm{N} / \mathrm{rad})$ & $u_{o}(\mathrm{~m} / \mathrm{sec})$ & $\mathrm{a}, \mathrm{b}(\mathrm{m})$ & $\mathrm{ds}(\mathrm{m})$ \\
\hline Nominal Value & 1573 & 2782 & $\begin{array}{c}46000(\mathrm{f}) \\
38850(\mathrm{r})\end{array}$ & 32 & $1.034,1.491$ & 1.9 \\
\hline
\end{tabular}

Figure 2 shows the magnitude plots of the frequency response from control (steering) to lateral tracking error. It can be seen that the $2 \mathrm{PPU}$ algorithm (preview time $=0.5$ seconds) demonstrated improved performance over standard 2P algorithm, while the 2PPW algorithm (preview time $=0.05$ seconds) significantly reduces the effectiveness of the control player. Figure 3 shows the frequency response of the transfer functions from steering to vehicle lateral acceleration. It can be seen that the 2PPU algorithm improves tracking error and achieves smoother ride quality (smaller lateral acceleration) simultaneously. The effect of preview action of the 2PPW algorithm, on the other hand, is deteriorated tracking error and ride quality simultaneously. This is in contrast to the fact that feedback-only algorithms can only trade-off between these two performance objectives.

\section{CONCLUSIONS}

Solutions of the continuous-time two-player game problems under preview information structures were presented. It was shown that when preview information is available, the game Riccati Equation is modified and needs to be solved with two partial differential equations simultaneously. A feedforward term is added to the optimal strategy, which takes advantage of the preview information, and reacts to the opponent's action ahead of time. When the preview time is zero, the preview two-player solutions reduce to the classical two-player game theory results. Frequency domain analysis results were used to demonstrate the effectiveness of the preview algorithms. It was found that the preview action significant improves the effectiveness of the advantaged player. 


\section{ACKNOWLEDGMENT}

This research was supported by the U.S. Army TARDEC under the contract DAAE0794-C-R094.

\section{REFERENCES}

Basar, T. and Bernhard, P., $H_{\infty}$-Optimal Control and Related Minimax Design Problems--A Dynamic Game Approach, 2nd edition, Birkhauser, 1995.

Dorato, P. and Drenick, R.F., "Optimality, Insensitivity, and Game Theory," in Sensitivity methods in control theory; proceedings of International Symposium on Sensitivity Analysis, 1964, Dubrovnik, Croatia.

Fenton, R.E., Melocik, G.C., and Olson, K.W., "On the Steering of Automated Vehicles: Theory and Experiment," IEEE Trans. on Automatic Control, Vol.AC-21, No.3, June 1976.

Kuhn, H.W., "Extensive Games and the Problem of Information," in Contributions to the Theory of Games, edited by H.W. Kuhn and A.W. Tucker, Annals of Mathematics Studies, No.28, Princeton University Press, 1953, pp.193-216

Ma, W. and Peng, H., "Worst-case Evaluation Methods for Vehicle Control Systems," Proceedings of the 1996 ASME International Congress and Exposition, Atlanta, GA, November 1996.

Mageirou, E.F. and Ho, Y.C., "Decentralized Stabilization via Game Theoretical Methods," Automatica, Vol.33, 1977, pp.393-399.

Peng, H. and Tomizuka, M., "Vehicle Lateral Control for Highway Automation," Proceedings of the American Control Conference, San Diego, USA, May, 1990.

Tomizuka, M. and Whitney, D.E., "Optimal Discrete Finite Preview Problems (Why and How is Future Information Important?)" Transactions of ASME Journal of Dynamic Systems, Measurement and Control, Dec. 1975, pp.319-325.

Tomizuka, M. "The Continuous Optimal Finite Preview Control Problem," Transactions of the Society of Instrument and Control Engineers, Vol.12, No.1, pp.7-12, Feb. 1976.

von Neumann, J. Morgenstern, O., Theory and Game and Economic Behavior, Princeton University Press, 2nd edition, 1947.

Witsenhausen, H.S., "A Minimax Control Problem for Sampled Linear Systems," IEEE Transactions for Automatic Control, AC-13:5-21, February 1968.

Yaesh, I. and Shaked, U., "Minimum $H_{\infty}$-norm Regulation of Linear Discrete-time Systems and its Relation to Linear Quadratic Difference Games," Proceedings of the 29th IEEE Conference of Decision and Control, 1989, pp.942-947. 


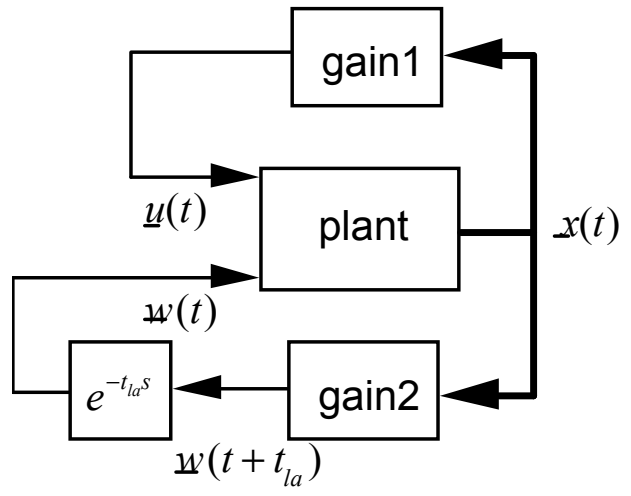

(a) control advantaged case

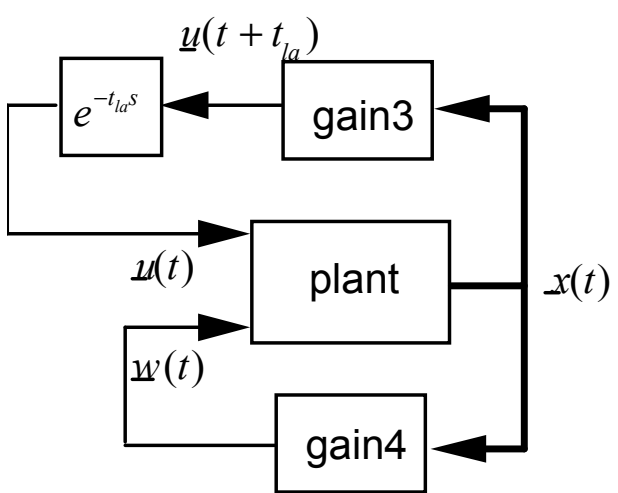

(b) disturbance advantaged case

Figure 1 Game problem with actuation delays

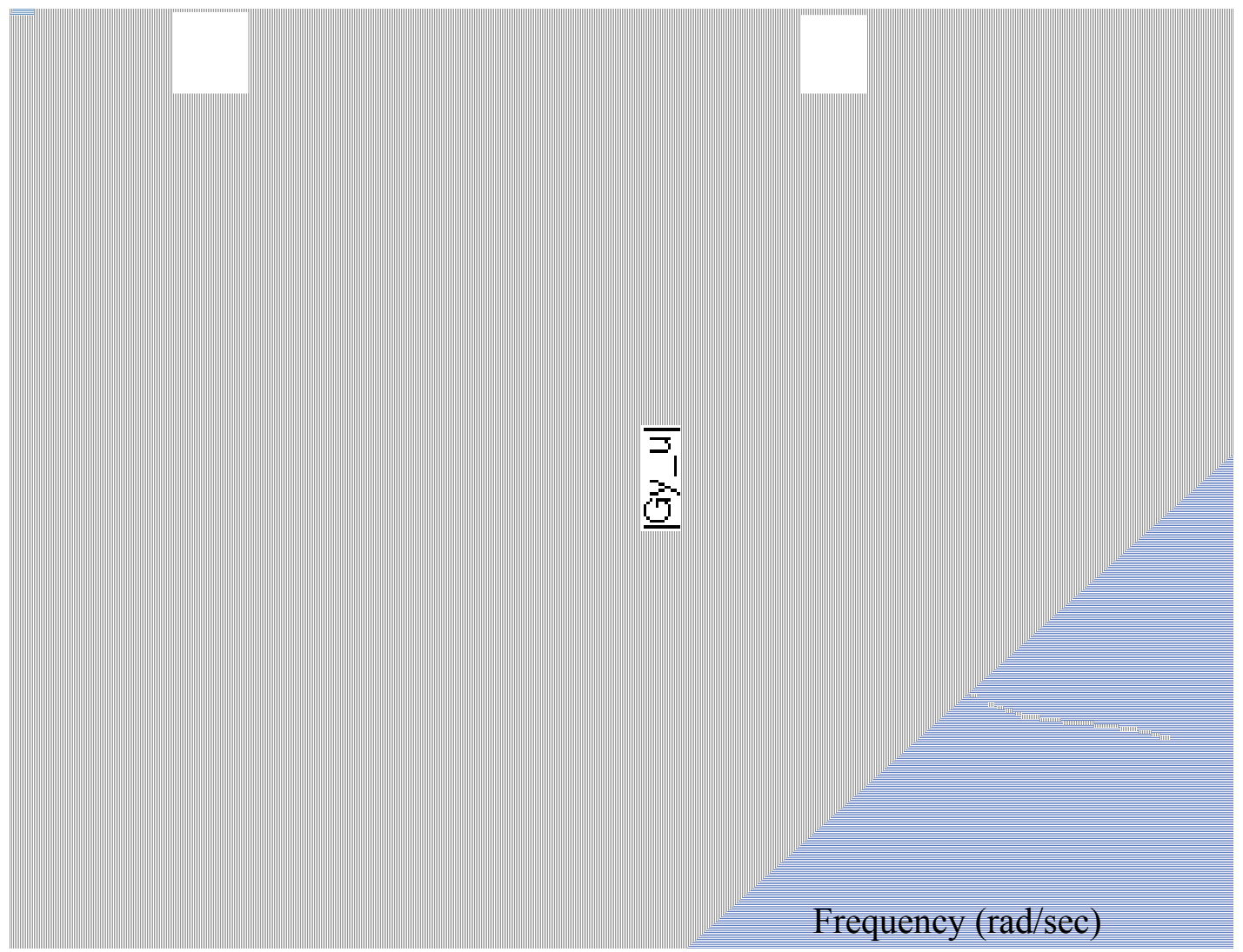

Figure 2 Frequency response of the lateral tracking error response 


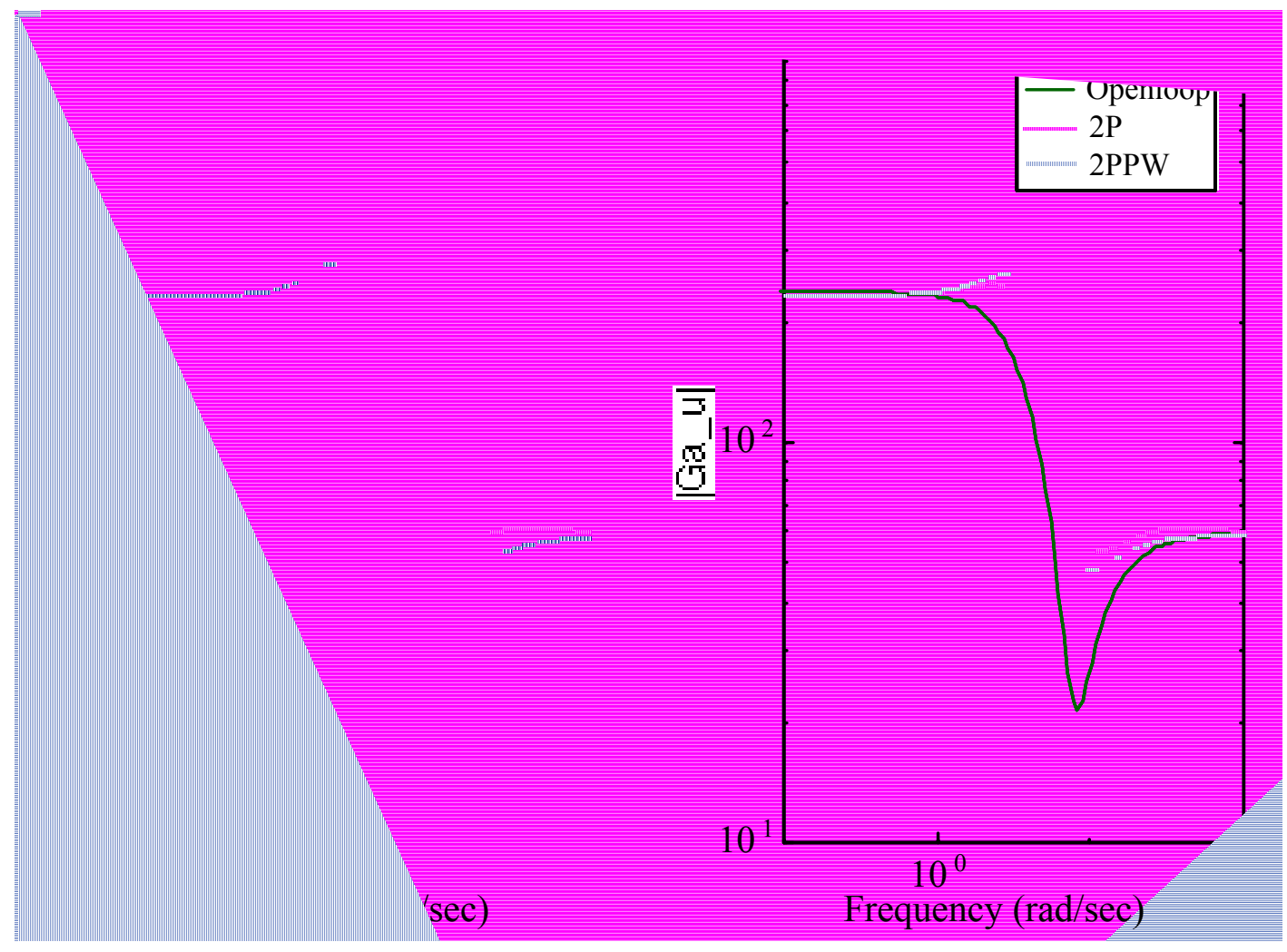

Figure 3 Frequency response of lateral acceleration 